Check for updates

Cite this: RSC Adv., 2019, 9, 35969

\title{
Effects of Bailing capsule on diabetic nephropathy based on UPLC-MS urine metabolomics
}

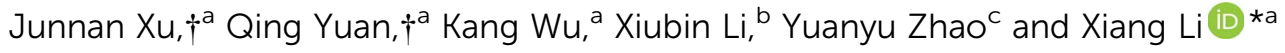

Diabetic nephropathy (DN) is one of the most common microvascular diabetes complications and has become a threat to human health. Bailing capsules (BLCs), containing fermentation products of Cordyceps sinensis, have been commonly used for treatment of renal dysfunction, such as DN. However, mechanisms underlying the protective effects of BLC remain largely obscure and await more investigation. In this study, UPLC-MS-based comprehensive metabolomics along with pattern recognition was applied to explore the urine metabolic alteration of DN as well as therapeutic mechanisms of BLC. Nineteen differentially expressed endogenous metabolites were identified related to DN, which were involved in the perturbations of tyrosine metabolism, tryptophan metabolism, glycine metabolism, purine metabolism, glutamine metabolism, phenylalanine metabolism, histidine metabolism and TCA cycle metabolism pathways. After drug intervention, most of the biomarkers exhibited a certain extent towards normal levels $(P<0.05)$, which indicated that BLC was an effective drug for treating DN and might play its therapeutic role by retrieving abnormal metabolism pathways. The data obtained in this research may pave the way for further exploration of DN and provide key clues to understand the protective effect of BLC.

Received 3rd July 2019

Accepted 23rd October 2019

DOI: $10.1039 / c 9 r a 05046 a$

rsc.li/rsc-advances
Chinese medicine. ${ }^{7}$ Since the Qing dynasty, it has been widely used as a health care product to replenish the kidney and soothe the lung for centuries. ${ }^{8}$ Recent pharmacological researches showed that Cordyceps sinensis has antioxidation activity, immunomodulating effect, anti-mutation effect and antitumour effect. ${ }^{9,10}$ Previous study also demonstrated that Cordyceps could protect against chronic kidney disease progression by affecting the TLR4/NF-KB lipid and redox signaling pathway. ${ }^{11}$ Bailing capsule (BLC), containing fermentation products of Cordyceps sinensis, has been commonly used for treatment of renal dysfunction, such as DN. However, mechanisms underlying the protective of BLC remain largely obscure and await more investigation.

Metabolomics is a modern strategy focused on low molecular weight endogenous metabolites to capture comprehensive biochemical events in biological systems by multiple analytical techniques. ${ }^{3,12,13}$ It is a powerful tool that can not only systematically search for key biomarkers for monitoring and diagnosing disease, but also offer insights into the mechanisms of pharmaceutical effects. ${ }^{14-17}$ Therefore it is beneficial to explore heterogeneous metabolic disorders such as DN and estimate the therapeutic effect of drugs.

In the present work, we performed a metabolomics study based on ultra performance liquid chromatography-mass spectrometry (UPLC-MS) to discriminate the potential biomarkers of DN. Then, we investigated the underlying therapeutic mechanisms of BLC on DN.

\footnotetext{
${ }^{a}$ Department of Urology, The Organ Transplant Institute of PLA, The 8th Medical Center of Chinese PLA General Hospital, Beijing 100091, China. E-mail: lixiang6666@hotmail.com

${ }^{b}$ The Organ Transplant Institute of PLA, The 8th Medical Center of Chinese PLA General Hospital, Beijing Key Laboratory of Immunology Regulatory and Organ Transplantation, Beijing 100091, China

'Department of Organ Transplantation, Shanghai Changzheng Hospital, Naval Medical University, Shanghai 200003, China

$\dagger$ These authors contributed equally to this work.
} 


\section{Materials and methods}

\subsection{Study populations}

The study protocol was approved by the Institutional Ethics Committee of the 8th Medical Center of Chinese PLA General Hospital and followed the tenets of the Declaration of Helsinki. With informed consent obtaining from all patients, we recruited 48 type 2 diabetes participants for monitoring renal function change. The following two groups were defined according to the clinical course and urinary albuminuria levels: 16 patients without albuminuria (control, albumin-tocreatinine (ACR) ratio $<30 \mathrm{mg} \mathrm{g}^{-1}$ ) and 32 patients with microalbuminuria (DN; $30<\mathrm{ACR}<300 \mathrm{mg} \mathrm{g}^{-1}$ ). Sixteen DN patients enrolled in the study were assigned to receive BLC treatment for 24 weeks as DN + BLC group. Sixteen clinically group-matched (age, sex, BMI, creatinine, baseline albuminuria status) DN subjects were selected as DN group. All patients received routine diabetes therapy scheme and diabetic diet. All urine samples were derived from $24 \mathrm{~h}$ urine collections and centrifuged ( $5000 \mathrm{~g}$ for $30 \mathrm{~min}$ at $4{ }^{\circ} \mathrm{C}$ ) to remove cell debris. The sample was then kept at $-80{ }^{\circ} \mathrm{C}$ for long-term storage.

\subsection{Reagents and materials}

HPLC-grade acetonitrile (ACN) and methanol were purchased from Merck (Darmstadt, Germany). All other reagents were purchased from Sigma-Aldrich (St. Louis, MO). Ultrapure water was purified with a Milli-Q purification system (Millipore, Billerica, MA). BLC was purchased from Huadong Medicine Company (Hangzhou, Zhejiang, China).

\subsection{Sample preparation}

To precipitate protein, four times of methanol (containing $0.01 \mathrm{mg} \mathrm{mL}{ }^{-1} \mathrm{~L}$-2-chlorophenylalanine as internal standard) was added to $100 \mu \mathrm{L}$ aliquots of urine in $1.5 \mathrm{~mL}$ centrifuge tube. The mixture was vortexed vigorously for $1 \mathrm{~min}$ and then centrifuged at $14000 \mathrm{~g}$ for $15 \mathrm{~min}$ at $4{ }^{\circ} \mathrm{C}$. After transferred to autosampler vial kept, $4 \mu \mathrm{L}$ of supernatant was injected for UPLC-MS analysis. Quality control (QC) was a mixture by pooling $10 \mu \mathrm{L}$ of each sample.

\subsection{Data acquisition}

Chromatographic analysis was performed on Agilent 1290 Infinity UPLC System (Palo Alto, California, USA). A Waters XSelect HSS T3 Column $(2.1 \mathrm{~mm} \times 100 \mathrm{~mm}, 2.5 \mu \mathrm{m}$, Milford, USA) was used for separation. The mobile phase consisted of solvent A ( $0.1 \%$ formic acid in water, v/v) and solvent B $(0.1 \%$ formic acid in acetonitrile, $\mathrm{v} / \mathrm{v}$ ). The gradient for chromatographic analysis was as follows: 0-2 $\mathrm{min}, 3 \% \mathrm{~B}$; 2-16 min, 330\% B; 16-18 min, 30-95\% B; 18-19.5 min, 95\% B; 19.5-20 min at $95-3 \% \mathrm{~B}$ and subsequently re-equilibrated for $4 \mathrm{~min}$. The flow rate was $0.4 \mathrm{~mL} \mathrm{~min}^{-1}$.

The mass range was set at $m / z 50-1000$ at $1 \mathrm{~Hz}$ in positive mode. The parameters were employed as follows: capillary voltage of $3.5 \mathrm{kV}$ and nebulizer pressure of 45 psig. The gas temperature was set to $350{ }^{\circ} \mathrm{C}$ with drying gas flow of 11
$\mathrm{L} \min ^{-1}$. The fragmentor voltage and skimmer voltage were set at $120 \mathrm{~V}$ and $60 \mathrm{~V}$, respectively. The MS/MS spectra of metabolites were obtained at 0.5 spectra per $\mathrm{s}$, with a medium isolation window $(\sim 4 \mathrm{~m} / \mathrm{z})$ and collision energy range from 10-30 eV.

\subsection{Data processing}

The LC-MS data was converted to mzData formats via Agilent MassHunter Qualitative software. The program XCMS was applied so as to nonlinear alignment of the data and automatic integrate and extract the peak intensities. The variables presenting in at least $80 \%$ of either group were extracted. ${ }^{\mathbf{1 8}}$ For further analysis, the data were normalized to the sum of all peak areas of each sample by MATLAB 7.0 software (The MathWorks, Inc, USA).

\subsection{Metabolomic data analysis}

To detect the potential biomarkers from different groups, the processed data were subject to partial least-squares discriminant analysis (PLS-DA) and orthogonal partial least-squares discriminant analysis (OPLS-DA) using SIMCA-P 11.0 (Umetrics, Sweden). As the variable importance in the projection (VIP) value obtained from the OPLS-DA model indicated the contribution to the classification, metabolites with VIP values were larger than 1.0 were selected for further Student's $t$ test. The quality of the OPLS-DA models was indicated by three parameters $\left(R^{2} X, R^{2} Y\right.$, and $\left.Q^{2} Y\right)$, in which they were employed to quantify the goodness-of-fit and to assess model predictability, respectively. ${ }^{\mathbf{1 9 , 2 0}}$

\subsection{Identification of biomarker metabolites}

The potential biomarkers were first identified by searching the experimental accurate mass and tandem mass spectra (MS/MS) in open-source database, such as METLIN (http:// www.metlin.scripps.edu), HMDB (http://www.hmdb.ca), the mass bank web site (http://www.massbank.jp/en/ database.html) and NIST database. Then, MS/MS and retention time (RT) of the potential biomarkers were further compared with those of reference standards.

\subsection{Statistical analysis}

The differences between groups were analyzed by One-way ANOVA with Tukey post hoc test on SPSS software. $P$ values < 0.05 were regarded as statistically significant.

\section{Results}

\subsection{Study population and samples}

A total of 16 type 2 diabetes controls and 32 DN patients from the 8th Medical Center of Chinese PLA General Hospital were screened. According to the clinical and demographic characteristics, the groups were well balanced (Table 1). We observed no significant difference in age, sex, BMI, creatinine, systolic $\mathrm{BP}$, diastolic BP and HbA1c (\%) among three groups. The DN group showed significantly lower eGFR, higher albuminuria, and higher urine ACR compared with the control group ( $p<$ 
Table 1 Clinical characteristics in 48 patients with type 2 diabetes mellitus $^{a}$

\begin{tabular}{|c|c|c|c|}
\hline & Control & DN & $\mathrm{DN}+\mathrm{BLC}$ \\
\hline Number & 16 & 16 & 16 \\
\hline Male/female & $3 / 13$ & $4 / 12$ & $4 / 12$ \\
\hline Age (years) & $59 \pm 7$ & $60 \pm 6$ & $61 \pm 6$ \\
\hline BMI $\left(\mathrm{kg} \mathrm{m}^{-2}\right)$ & $28 \pm 4$ & $28 \pm 4$ & $28 \pm 4$ \\
\hline Creatinine $\left(\mathrm{mg} \mathrm{dL}^{-1}\right)$ & $0.8 \pm 0.1$ & $0.9 \pm 0.1$ & $0.8 \pm 0.1$ \\
\hline Systolic BP (mmHg) & $119 \pm 10$ & $127 \pm 19$ & $124 \pm 17$ \\
\hline Diastolic BP (mmHg) & $74 \pm 11$ & $79 \pm 13$ & $77 \pm 12$ \\
\hline HbA1c $(\%)$ & $6.2 \pm 0.8$ & $6.7 \pm 1.1$ & $6.5 \pm 1.0$ \\
\hline Diabetes duration (years) & $10 \pm 3$ & $15 \pm 4$ & $14 \pm 3$ \\
\hline $\mathrm{eGFR}\left(\mathrm{mL} \min ^{-1}\right)$ & $93 \pm 21$ & $79 \pm 20^{b}$ & $88 \pm 23$ \\
\hline Albuminuria $\left(\mathrm{mg} \mathrm{L}^{-1}\right)$ & $0.7 \pm 0.4$ & $8 \pm 5^{b}$ & $4 \pm 3^{c}$ \\
\hline Urine ACR $\left(\mathrm{mg} \mathrm{g}^{-1}\right)$ & $5 \pm 2$ & $108 \pm 46^{b}$ & $44 \pm 22^{c}$ \\
\hline
\end{tabular}

${ }^{a} \mathrm{DN}$, diabetic nephropathy; BLC, Bailing capsule; eGFR, estimated glomerular filtration rate. ACR: albumin to creatinine ratio. Data reported as mean \pm standard deviation (SD). ${ }^{b}$ Significantly altered compared to control group. ${ }^{c}$ Significantly $(p<0.05)$ altered compared to DN group.

0.05), while there was no significant difference in eGFR between the DN and DN + BLC group. Despite matching for baseline albuminuria stage, a significant difference in albuminuria levels between DN + BLC group and DN group was observed, suggesting that BLC had a protective effect in DN. In addition, urine albumin to creatinine ratio (ACR) was statistically lower in $\mathrm{DN}+\mathrm{BLC}$ subjects than in DN group $(p<0.01)$, which also indicating that BLC had a therapeutic influence in DN (Table 1).

\subsection{Metabolic profiles of controls and DN patients}

OPLS-DA method was performed to achieve maximum separation between groups and screen out differential metabolites from a huge amount of signals. Score plot of the supervised OPLS-DA model (Fig. 1) displayed that DN group and control group were entirely isolated and distinctly separated in the first dimension, indicating that there were significant metabolic abnormalities in the DN patients. The goodness-of-fit and model predictability of the OPLS-DA models was quantified by three parameters $\left(R^{2} X, R^{2} Y\right.$, and $\left.Q^{2} Y\right)$. The performance statistics of $R^{2} X, R^{2} Y$ and predictive parameter $Q^{2} Y$ were $0.617,0.992$ and 0.708 respectively, indicating that the model was valid and prediction.

The corresponding $S$-plots and VIPs of OPLS-DA model were then created to screen out the potential metabolites that were responsible for the differences. Based on VIP threshold (VIP $>1$ ) in OPLS-DA model and Student's $t$ test $(p<0.05)$, nineteen differentially expressed metabolites enabling the separation between control group and DN group, were marked as potential biomarkers. The detailed information of these biomarkers summarized in Table 2.

\subsection{Effect of BLC treatment}

The therapeutic effect of BLC in DN was confirmed as exhibited in the clinical characteristics alteration, including albuminuria and urine ACR. To further reveal the mechanisms of BLC, 19 significantly altered metabolites connected with DN were regarded as the monitoring indicators to establish model for analysis. This newly built PLS-DA model was established to further uncover the metabolic alterations between these groups. The data of the PLS-DA model showed that $R^{2} Y=0.801$ and $Q^{2}$ (cum) $=0.739$, which suggested the model was robust and reliable. As shown in Fig. 2, a distinct separation of the DN group and control group was obtained, while the BLC treatment group had the tendency to resume to the control group, demonstrating that BLC exhibited a certain extent of treatment effect on DN. The results suggested that the metabolic profiling was ameliorated with the BLC therapy, which could modify the dysregulated metabolites caused by DN and return them to normal levels.

Furthermore, one-way ANOVA analysis was accomplished among the three groups to evaluate the recovery situation of the altered metabolites by treatment with BLC (Table 2). The relative peak intensity of the 19 potential biomarkers is shown in
A

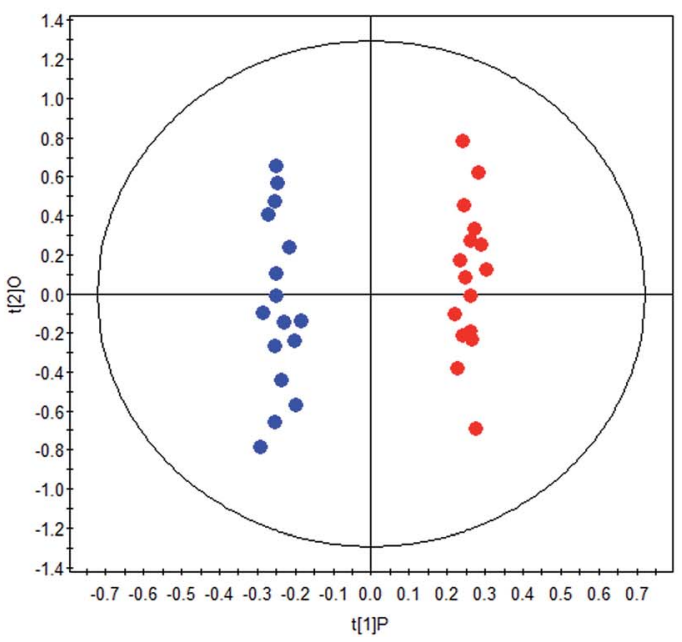

B

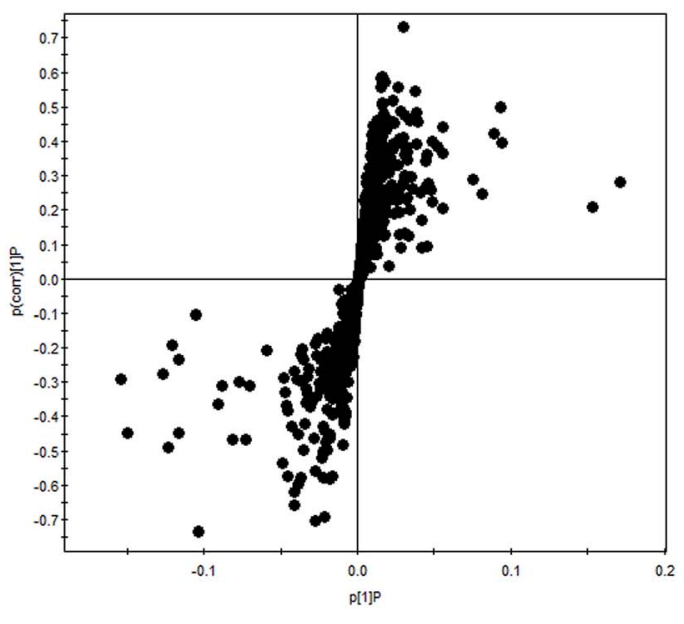

Fig. 1 OPLS-DA scores plot (A) and S-plot (B) of control group (•) and DN group (๑). 
Table 2 Metabolites discriminating DN and type 2 diabetes subjects by UPLC-MS analysis ${ }^{a}$

\begin{tabular}{|c|c|c|c|c|c|c|}
\hline & & & DN vs. control & $\begin{array}{l}\mathrm{DN}+ \\
\mathrm{BLC} v s . \mathrm{DN}\end{array}$ & & \\
\hline Metabolites & Trend $^{b}$ & Related pathway & $P$ value ${ }^{c}$ & FC & $P$ value $^{c}$ & FC \\
\hline 5-Methoxytryptophan & $\downarrow$ & Tryptophan metabolism & $5.79 \times 10^{-3}$ & 0.46 & $4.82 \times 10^{-3}$ & 2.70 \\
\hline$p$-Hydroxyphenylacetic acid & $\uparrow$ & Phenylalanine metabolism & $8.01 \times 10^{-4}$ & 3.89 & $8.24 \times 10^{-2}$ & 0.65 \\
\hline 5-Hydroxy-6-methoxyindole glucuronide & $\uparrow$ & Tryptophan metabolism & $8.09 \times 10^{-3}$ & 2.22 & $3.87 \times 10^{-3}$ & 0.33 \\
\hline Indoxyl glucuronide & $\uparrow$ & Tryptophan metabolism & $2.75 \times 10^{-2}$ & 2.72 & $5.47 \times 10^{-3}$ & 0.50 \\
\hline L-Histidine & $\downarrow$ & Histidine metabolism & $6.80 \times 10^{-3}$ & 0.38 & $6.10 \times 10^{-3}$ & 3.04 \\
\hline Uric acid & $\uparrow$ & Purine metabolism & $3.32 \times 10^{-4}$ & 2.87 & $2.75 \times 10^{-2}$ & 0.39 \\
\hline 2-Phenylacetamide & $\uparrow$ & Phenylalanine metabolism & $3.82 \times 10^{-2}$ & 2.01 & $9.16 \times 10^{-2}$ & 0.82 \\
\hline Phenyl glucuronide & $\uparrow$ & & $8.31 \times 10^{-3}$ & 2.50 & $1.00 \times 10^{-2}$ & 0.47 \\
\hline Purine & $\uparrow$ & Purine metabolism & $3.90 \times 10^{-2}$ & 1.86 & $8.88 \times 10^{-2}$ & 0.59 \\
\hline Indoxylsulfuric acid & $\uparrow$ & Tryptophan metabolism & $4.29 \times 10^{-2}$ & 2.14 & $2.26 \times 10^{-2}$ & 0.62 \\
\hline$p$-Cresol sulfate & $\uparrow$ & Tyrosine metabolism & $1.93 \times 10^{-2}$ & 1.56 & $3.10 \times 10^{-2}$ & 0.55 \\
\hline$p$-Cresol glucuronide & $\uparrow$ & Tyrosine metabolism & $3.92 \times 10^{-3}$ & 3.44 & $1.45 \times 10^{-2}$ & 0.48 \\
\hline Citric acid & $\downarrow$ & TCA cycle & $9.53 \times 10^{-3}$ & 0.47 & $1.47 \times 10^{-2}$ & 2.25 \\
\hline
\end{tabular}

${ }^{a}$ Abbreviations: DN, diabetic nephropathy; BLC, Bailing capsule; FC, fold change. ${ }^{b}$ Arrow $(\uparrow)$ indicates relative increase in signal. Arrow $(\downarrow)$ indicates relative decrease in signal. ${ }^{c}$ The $P$ value was calculated by one-way ANOVA and then by the Tukey post hoc test for comparisons of multiple groups.

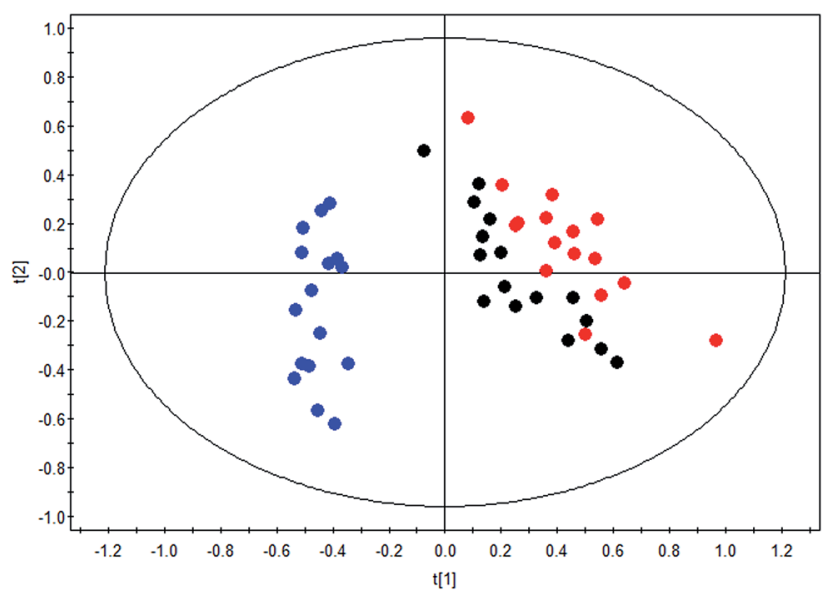

Fig. 2 PLS-DA scores plot of nineteen metabolites from control group (๑), DN group (๑) and DN + BLC group (๑).

Fig. 3. Following BLC treatment, the levels of fourteen metabolites including 5-methoxytryptophan, 5-hydroxy-6methoxyindole glucuronide, 2-phenylglycine, indoxyl glucuronide, L-histidine, uric acid, phenyl glucuronide, L-tryptophan, hippuric acid, 4-hydroxyhippuric acid, indoxylsulfuric acid, $p$ cresol sulfate, $p$-cresol glucuronide and citric acid were obviously improved in DN + BLC group in comparison with DN group. The other five metabolites were also ameliorated to some degrees. Then, overview of the metabolites and major metabolic pathways changes in DN was built by exploring KEGG PATHWAY Database (Fig. 4). All the results above indicated that the protective effect of BLC on DN may act by partially resuming the disequilibrium of tyrosine metabolism, tryptophan metabolism, glycine metabolism, purine metabolism, glutamine metabolism, phenylalanine metabolism, histidine metabolism and TCA cycle metabolism pathways.

\section{Discussion}

The local and traditional uses of Cordyceps sinensis are replenishing the kidney and soothing the lung. ${ }^{8}$ Recent pharmacological stated that it possesses widely pharmacological activities, including kidney protection, immunoregulation, antioxidation and anti-cancer., ${ }^{\mathbf{9 1 0}}$ Bailing capsule (BLC), containing fermentation products of Cordyceps sinensis, has been commonly used for treatment of renal dysfunction. ${ }^{\mathbf{1 1}}$ But the mechanisms underlying the protective of BLC are still largely unknown. In our study, metabolomics investigation combined with OPLS-DA analysis was used to discriminate the potential biomarkers of DN and investigate the underlying therapeutic mechanisms of BLC. Nineteen potential biomarkers were identified, which were associated with tyrosine metabolism, tryptophan metabolism, glycine metabolism, purine metabolism, glutamine metabolism, phenylalanine metabolism, histidine metabolism and TCA cycle metabolism pathways. Based on the alteration of biomarkers after BLC treatment, it was confirmed that BLC might play its therapeutic role by retrieving abnormal metabolism pathways.

A distinct tryptophan metabolism was observed in DN group, in which six metabolites were significantly altered. Among them, L-tryptophan and 5-methoxytryptophan were significantly 


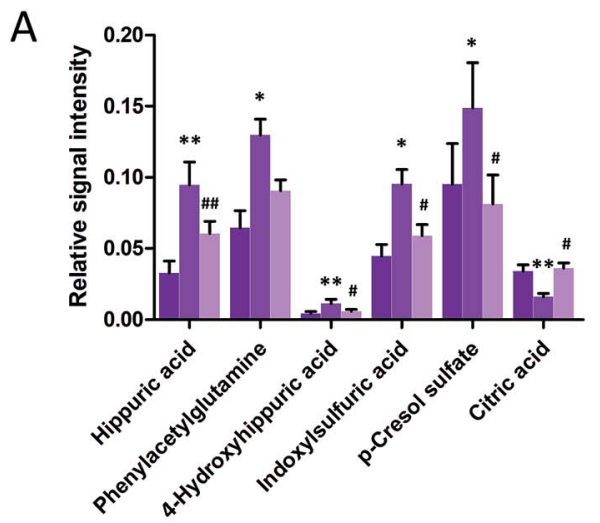

\section{B}
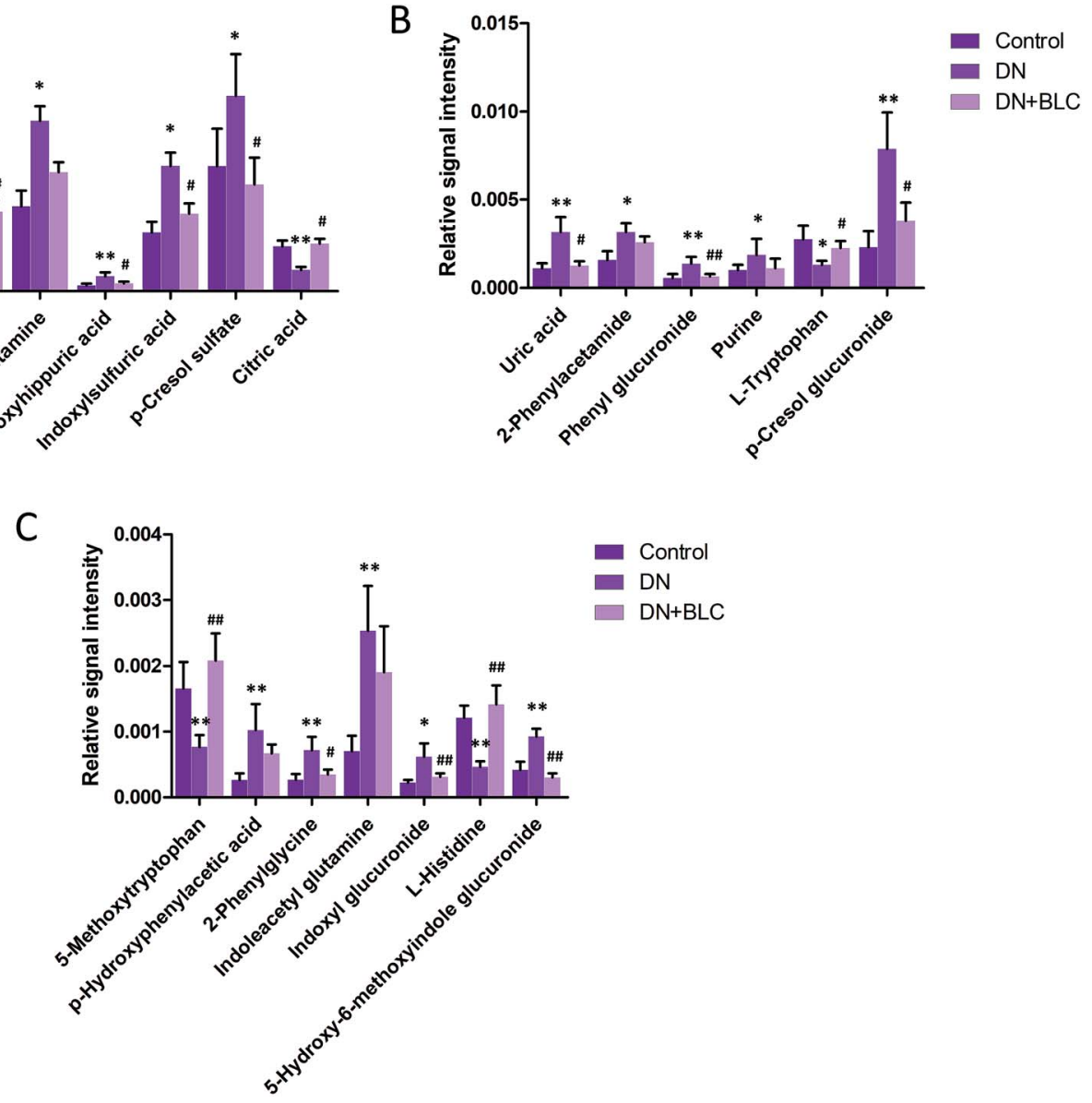

Fig. 3 Bar plots show UPLC-MS relative signal intensities for candidate metabolites (A-C) in control, DN and DN + BLC groups. Data are expressed as mean \pm S.D. (DN vs. control group, $* p<0.05$, ** $p<0.01$; DN + BLC vs. DN group, $\# p<0.05, \# \#<0.01$ ).

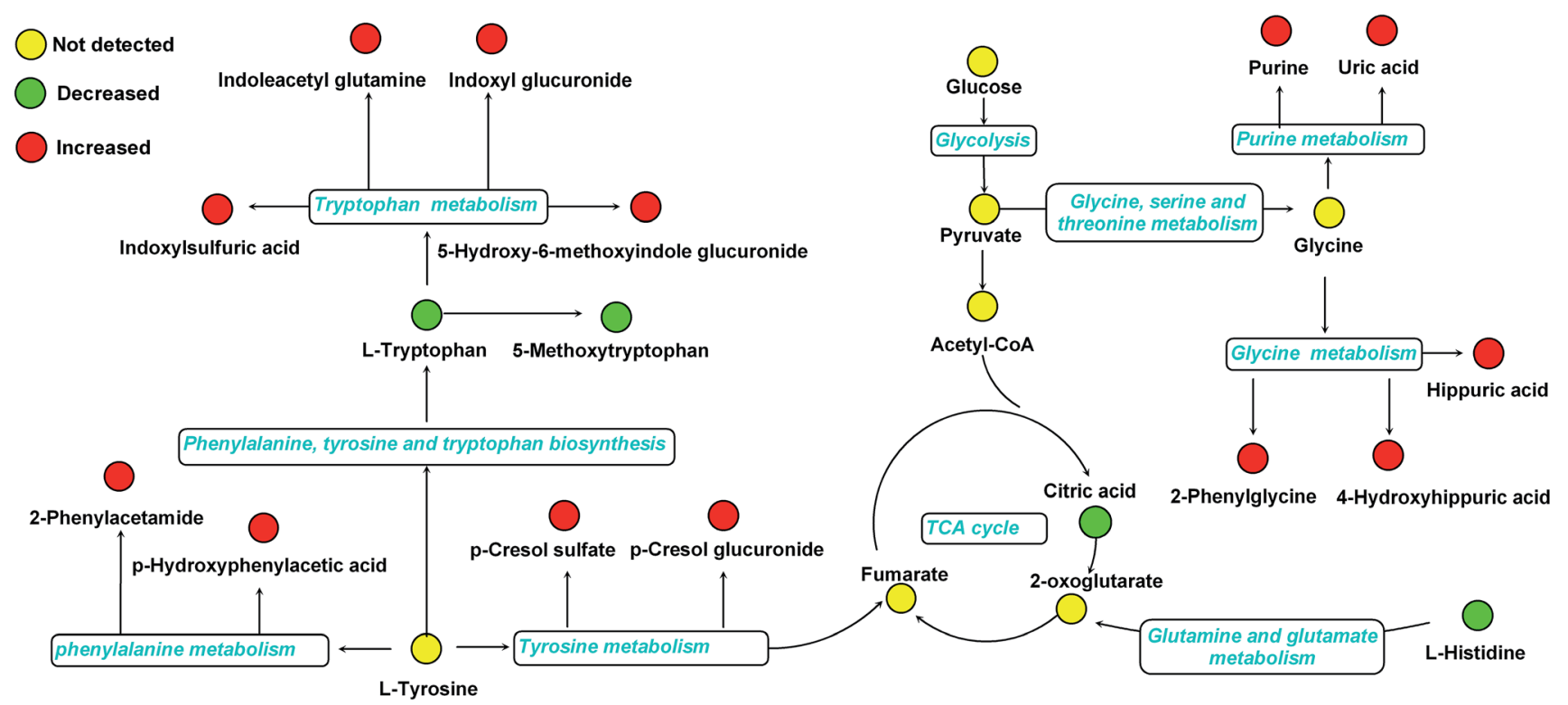

Fig. 4 Structure of metabolism pathway networks involved in DN patients. The metabolites are shown in color: red represents increased metabolite, green represents decreased metabolites compared to controls and yellow represents no detected metabolites, respectively. The blue italic words are pathway's names. 
decreased in DN patients compared to the controls, while, 5hydroxy-6-methoxyindole glucuronide, indoleacetyl glutamine, indoxyl glucuronide and indoxylsulfuric acid were significantly elevated. Increasing evidence showed that disturbed tryptophan metabolism was associated with the decline in eGFR and tryptophan related metabolites could be potential prognostic markers for $\mathrm{DN}^{.21-23}$ The present investigation added new evidence that tryptophan metabolism is a key metabolic pathway in the progression of $\mathrm{DN}$. As a rate-limiting enzyme in tryptophan catabolism, indoleamine 2,3-dioxygenase was a key player in immunoregulatory of chronic inflammation and renal injury.,24 It was reported that indoleamine 2,3-dioxygenase activity was induced in patients with type $2 \mathrm{DN}$, which may lead to the decreased level of tryptophan and increased level of oxidation metabolites of tryptophan in DN. Meanwhile, it has been noticed that several glucuronides, such as 5-hydroxy-6methoxyindole glucuronide, indoxyl glucuronide, phenyl glucuronide and $p$-cresol glucuronide, were significantly upregulated in the patients with DN, which was in accordance with previous research. ${ }^{25}$ Since glucuronidation could assist the excretion of toxic substances, the elevated glucuronides might reflect the process of toxins elimination when kidney was injured. After BLC treatment, five of the six tryptophan metabolites and all glucuronides were dramatically revised and restored to normal levels, which indicated that BLC may play its protective role in DN through rebalancing tryptophan metabolism and reducing glucuronides.

In our differentially expressed metabolites, $p$-cresol sulfate and $p$-cresol glucuronide belonging to the tyrosine metabolism were remarkably elevated in patients with DN. It was reported that $p$-cresol sulfate was inversely related to eGFR in a large population base cohort with minimal renal function decline and could be early markers of renal function decline, ${ }^{26}$ which indirectly supported our results. Cresols are generated endogenously from tyrosine metabolism by microflora in colon. ${ }^{27}$ Obvious imbalanced tyrosine and tryptophan metabolism indicated that microbial metabolism of amino acids in intestinal flora played an important role in DN. In DN + BLC group, both of the cresols were significantly decreased and had the tendency to resume to normal levels, which suggested that BLC may relieve DN by retrieving the gut microbial and repairing abnormal tyrosine metabolism pathway.

Interestingly, several uremic toxins, including hippuric acid, 4-hydroxyhippuric acid, indoxylsulfuric acid and $p$-cresol sulfate, were significantly upregulated in DN patients. Earlier finding showed that serum uremic toxins have a significant role in the vascular disease and higher mortality observed in chronic kidney disease patients. ${ }^{28}$ Moreover, a recent research demonstrated that uremic toxins, such as indoxyl sulfate and $p$-cresol sulfate, directly promote vascular calcification via activation of inflammation and coagulation pathways and were strongly associated with impaired glucose homeostasis, ${ }^{29}$ which partially explained the phenomenon of elevated uremic toxins observed in our study. In general, main elimination pathway of uremic toxins was via urinary excretion with active tubular secretion. ${ }^{30}$ However, when DN occurred and renal function was compromised, elimination of uremic toxins became impaired, which lead to increased levels of uremic toxins in urine. After BLC administration, the levels of all abnormal uremic toxins remarkably declined and exhibited a certain degree of normalization, which indicated that reducing accumulation of uremic toxins may play an important role in BLC's therapeutic effects.

The levels of uric acid and purine were found to be significantly higher in urine of DN patients, which were mapped into purine metabolism. As final enzymatic product in the degradation of purine nucleosides, it has been reported uric acid is involved in various stages of DN onset and progression. ${ }^{31}$ In the meantime, there is evidence that purine metabolism related metabolites including xanthine, xanthosine, guanosine and hypoxanthine were upregulated in DN and diabetic patients, ${ }^{32,33}$ which was in agreement with our results. It was also reported that an overactivated purine metabolism was observed in DN and it could be normalized by the specific mitochondrial complex-1 inhibitor, which supported our results. ${ }^{34}$ Purine metabolism has been shown to increase the activation of mitochondrial defense mechanisms under oxidative stress conditions, such as diabetes and renal injury. ${ }^{33}$ In addition, a recent study found that the overactivation of mitochondrial activity in the kidney could contribute to metabolic disorders and the pathogenesis of early DN. Therefore, the increased uric acid and purine in DN patients may be related to mitochondrial antioxidant defense processes. It was observed that concentration of uric acid and purine return to normal after BLC treatment, which suggested that BLC may attenuate $\mathrm{DN}$ by rebalancing the purine metabolism pathway.

\section{Conclusion}

In summary, comprehensive metabolomics along with pattern recognition was applied to explore the metabolic alteration of DN as well as therapeutic mechanisms of BLC. Nineteen differentially expressed endogenous metabolites were identified related to $\mathrm{DN}$, which were involved in the perturbations of tyrosine metabolism, tryptophan metabolism, glycine metabolism, purine metabolism, glutamine metabolism, phenylalanine metabolism, histidine metabolism and TCA cycle metabolism. After drug intervention, most of the biomarkers exhibited a certain extent towards normal levels $(P<0.05)$, which indicated that BLC was an effective drug for treating $\mathrm{DN}$ and might play its therapeutic role by retrieving abnormal metabolism pathways. The data obtained in this research may pave the way for further exploration of DN and provide key clues to understand the protective effect of BLC. In the future, we will try to discover the underlying mechanism regarding how BLC regulates the disordered metabolism pathways.

\section{Conflicts of interest}

The authors declare no conflict of interest. 


\section{Acknowledgements}

This research was supported by National Natural Science Foundation of China (81800660, 81770745, 81570679, 81200547) and Beijing NOVA program (Z161100004916141).

\section{References}

1 M. Lv, Z. Chen, G. Hu and Q. Li, Drug Discovery Today, 2015, 20, 332-346.

2 X. Xiang, H.-D. Cai, S.-l. Su, X.-x. Dai, Y. Zhu, J.-m. Guo, H. Yan, S. Guo, W. Gu, D.-w. Qian, Z.-s. Tang and J.-a. Duan, Pharmacol. Res., 2019, 139, 26-40.

3 M. J. Pena, H. J. L. Heerspink, M. E. Hellemons, T. Friedrich, G. Dallmann, M. Lajer, S. J. L. Bakker, R. T. Gansevoort, P. Rossing, D. Zeeuw and S. S. Roscioni, Diabetic Med., 2014, 31, 1138-1147.

4 Y. Liu, X. Chen, Y. Liu, T. Chen, Q. Zhang, H. Zhang, Z. Zhu, Y. Chai and J. Zhang, Chem.-Biol. Interact., 2019, 108815, DOI: 10.1016/j.cbi.2019.108815.

5 J. Brenneman, J. Hill and S. Pullen, Bioorg. Med. Chem. Lett., 2016, 26, 4394-4402.

6 K. M. Sas, A. Karnovsky, G. Michailidis and S. Pennathur, Diabetes, 2015, 64, 718-732.

7 X. Li, F. Wang, Q. Liu, Q. Li, Z. Qian, X. Zhang, K. Li, W. Li and C. Dong, BMC Genomics, 2019, 20, 337.

8 H. Zhang, Y. Li, J. Mi, M. Zhang, Y. Wang, Z. Jiang and P. Hu, Molecules, 2017, 22, 1800.

9 M. G. Shashidhar, P. Giridhar, K. Udaya Sankar and B. Manohar, J. Funct. Foods, 2013, 5, 1013-1030.

10 X. Zhou, Z. Gong, Y. Su, J. Lin and K. Tang, J. Pharm. Pharmacol., 2009, 61, 279-291.

11 T. Sun, W. Dong, G. Jiang, J. Yang, J. Liu, L. Zhao and P. Ma, Oxid. Med. Cell. Longevity, 2019, 2019, 16.

12 Y. Liu, Z. Hong, G. Tan, X. Dong, G. Yang, L. Zhao, X. Chen, Z. Zhu, Z. Lou, B. Qian, G. Zhang and Y. Chai, Int. J. Cancer, 2014, 135, 658-668.

13 R. O. Bahado-Singh, R. Akolekar, R. Mandal, E. Dong, J. Xia, M. Kruger, D. S. Wishart and K. Nicolaides, Am. J. Obstet. Gynecol., 2013, 208, 371.

14 Y.-H. You, T. Quach, R. Saito, J. Pham and K. Sharma, J. Am. Soc. Nephrol., 2016, 27, 466-481.

15 K. Sharma, B. Karl, A. V. Mathew, J. A. Gangoiti, C. L. Wassel, R. Saito, M. Pu, S. Sharma, Y.-H. You, L. Wang, M. DiamondStanic, M. T. Lindenmeyer, C. Forsblom, W. Wu, J. H. Ix, T. Ideker, J. B. Kopp, S. K. Nigam, C. D. Cohen, P.-H. Groop, B. A. Barshop, L. Natarajan, W. L. Nyhan and R. K. Naviaux, J. Am. Soc. Nephrol., 2013, 24, 1901-1912.

16 Y. Liu, S. Lu, L. Zhao, X. Dong, Z. Zhu, Y. Jin, H. Chen, F. Lu, Z. Hong and Y. Chai, Anal. Bioanal. Chem., 2018, 410, 33253335 .
17 M. Zhang, Y. Liu, M. Liu, B. Liu, N. Li, X. Dong, Z. Hong and Y. Chai, Metabolomics, 2019, 15, 13.

18 S. Bijlsma, I. Bobeldijk, E. R. Verheij, R. Ramaker, S. Kochhar, I. A. Macdonald, B. van Ommen and A. K. Smilde, Anal. Chem., 2005, 78, 567-574.

19 S. Wiklund, E. Johansson, L. Sjöström, E. J. Mellerowicz, U. Edlund, J. P. Shockcor, J. Gottfries, T. Moritz and J. Trygg, Anal. Chem., 2008, 80, 115-122.

20 E. S. Ong, L. Zou, S. Li, P. Y. Cheah, K. W. Eu and C. N. Ong, Mol. Cell. Proteomics, 2010, DOI: 10.1074/mcp.m900551mcp200.

21 C. A. Chou, C. N. Lin, D. T. Y. Chiu, I. W. Chen and S. T. Chen, J. Diabetes Invest., 2018, 9, 366-374.

22 F. M. van der Kloet, F. W. A. Tempels, N. Ismail, R. van der Heijden, P. T. Kasper, M. Rojas-Cherto, R. van Doorn, G. Spijksma, M. Koek, J. van der Greef, V. P. Mäkinen, C. Forsblom, H. Holthöfer, P. H. Groop, T. H. Reijmers and T. Hankemeier, Metabolomics, 2012, 8, 109-119.

23 E. P. Rhee, A. Souza, L. Farrell, M. R. Pollak, G. D. Lewis, D. J. R. Steele, R. Thadhani, C. B. Clish, A. Greka and R. E. Gerszten, J. Am. Soc. Nephrol., 2010, 21, 1041-2051.

24 P. Puccetti and U. Grohmann, Nat. Rev. Immunol., 2007, 7, 817.

25 T. Zhao, H. Zhang, T. Zhao, X. Zhang, J. Lu, T. Yin, Q. Liang, Y. Wang, G. Luo, H. Lan and P. Li, J. Pharm. Biomed. Anal., 2012, 60, 32-43.

26 C. Barrios, M. Beaumont, T. Pallister, J. Villar, J. K. Goodrich, A. Clark, J. Pascual, R. E. Ley, T. D. Spector, J. T. Bell and C. Menni, PLoS One, 2015, 10, e0134311.

27 K. K. Pasikanti, K. Esuvaranathan, Y. Hong, P. C. Ho, R. Mahendran, L. Raman Nee Mani, E. Chiong and E. C. Y. Chan, J. Proteome Res., 2013, 12, 3865-3873.

28 F. C. Barreto, D. V. Barreto, S. Liabeuf, N. Meert, G. Glorieux, M. Temmar, G. Choukroun, R. Vanholder and Z. A. Massy, Clin. J. Am. Soc. Nephrol., 2009, 4, 1551-1558.

29 B. Opdebeeck, S. Maudsley, A. Azmi, A. De Maré, W. De Leger, B. Meijers, A. Verhulst, P. Evenepoel, P. C. D'Haese and E. Neven, J. Am. Soc. Nephrol., 2019, 30, 751-766.

30 Y. Tsutsumi, T. Deguchi, M. Takano, A. Takadate, W. E. Lindup and M. Otagiri, J. Pharmacol. Exp. Ther., 2002, 303, 880-887.

31 D. I. Jalal, D. M. Maahs, P. Hovind and T. Nakagawa, Semin. Nephrol., 2011, 31, 459-465.

32 S. Kalim, C. B. Clish, J. J. Deferio, G. Ortiz, A. S. Moffet, R. E. Gerszten, R. Thadhani and E. P. Rhee, BMC Nephrol., 2015, 16, 98.

33 C.-J. Chen, W.-L. Liao, C.-T. Chang, Y.-N. Lin and F.-J. Tsai, J. Proteome Res., 2018, 17, 3997-4007.

34 M. Wu, S. Li, X. Yu, W. Chen, H. Ma, C. Shao, Y. Zhang, A. Zhang, S. Huang and Z. Jia, Am. J. Physiol. Renal. Physiol., 2019, 317, F593-F605. 